\title{
Prevalence and Location of Endplate Fracture and Subsidence after Oblique Lumbar Interbody Fusion for Adult Spinal Deformity
}

Gen Inoue ( $\nabla$ ginoue@kitasato-u.ac.jp )

Kitasato University School of Medicine

Wataru Saito

Kitasato University School of Medicine

Masayuki Miyagi

Kitasato University School of Medicine

Takayuki Imura

Kitasato University School of Medicine

\section{Eiki Shirasawa}

Kitasato University School of Medicine

\section{Yusuke Mimura}

Kitasato University School of Medicine

Akiyoshi Kuroda

Kitasato University School of Medicine

Yuji Yokozeki

Kitasato University School of Medicine

Tsutomu Akazawa

St. Marianna University School of Medicine

Toshiyuki Nakazawa

Kitasato University School of Medicine

Kentaro Uchida

Kitasato University School of Medicine

Masashi Takaso

Kitasato University School of Medicine

\section{Research Article}

Keywords: endplate fracture, subsidence, oblique lumbar interbody fusion, adult spinal deformity, corrective surgery

Posted Date: May 5th, 2021 
DOl: https://doi.org/10.21203/rs.3.rs-453128/v1

License: (c) (1) This work is licensed under a Creative Commons Attribution 4.0 International License. Read Full License 


\section{Abstract}

Background: Recently, Oblique lumbar interbody fusion (OLIF) is commonly indicated to correct the sagittal and coronal alignment in adult spinal deformity (ASD). Endplate fracture during surgery is one of major complication of OLIF, but the detailed location of fracture in vertebral endplate has not yet evaluated in ASD. We conducted this study to evaluate the incidence and location of endplate fracture and subsidence of the OLIF cage, and its impact on fusion status and alignment in ASD surgery.

Methods: 75 levels in 27 patients were analyzed using multiplanar CT to detect the endplate fracture immediately after surgery and subsidence at postoperative 1 year. The prevalence was compared between anterior and posterior, approach side and non-approach side, and concave and convex side. Their impact on fusion status, local and global alignment, and complication was also investigated.

Results: Endplate fracture was observed in 64 levels (85.3\%) in all 27 patients, and the incidence was significantly higher in posterior area compared with anterior area $(85.3 \%$ vs. $68.0 \%, p=0.02)$ of affected vertebra in sagittal plane. On the coronal plane, there was no significant difference in incidence between left (approach) side and right (non-approach) side (77.3\% and $81.3 \%$, respectively), or concave and convex side $(69.4 \%$ and $79.6 \%)$ of wedged vertebra. On the other hand, cage subsidence at postoperative one year was noted in $18.7 \%$ (14/75 levels), which was not associated with endplate fracture. Fusion status, local and global alignment, or complications were not associated with endplate fracture and subsidence.

Conclusion: Endplate fracture during OLIF procedure in ASD cases is hardly avoidable, possibly induced by corrective maneuver with ideal rod counter and cantilever force, but impacts less on subsequent cage subsidence, fusion status, and sustainment of corrected alignment in long fusion surgery performed even for elderly patients.

\section{Introduction}

A current trend in spinal fusion surgery is lateral lumbar interbody fusion (LLIF), which uses a minimally invasive lateral retroperitoneal transpsoas or anteropsoas approach to achieve interbody fusion with fewer complications [1-4]. An oblique lumbar interbody fusion (OLIF) approach to lumbar discs via the space between the aorta and left-sided psoas major, avoids damage to the neural structures and psoas, and corrects sagittal and coronal alignment found in adult spinal deformity (ASD) when combined with long-level posterior spinal fusion (PSF) [5-7].

Despite its potential for correction, endplate fracture during surgery is a complication of OLIF. Endplate fracture after OLIF procedure has been reported at a rate of $2.2-14.6 \%[4,8-10]$. Endplate fractures possibly result in cage subsidence, which a recent meta-analysis indicated at 5.1-12.2\% after OLIF [4, 9, 10]. Cage subsidence after OLIF is associated with greater age and body mass index (BMI), but overall fusion rate using autologous iliac crest bone was $98.4 \%$ and was not associated with cage subsidence [11]. However, the relationship between endplate fracture or subsidence and fusion state or sustainment 
of alignment correction after OLIF for ASD remains unclear. In the present study, we used data from multiplanar computed tomography (CT) reconstruction to determine the prevalence and location of endplate fracture and subsidence after OLIF corrective surgery for ASD, and their association with intervertebral fusion at 1 year postoperatively and spinopelvic and global alignment at a mean of 47 months.

\section{Materials And Methods}

\section{Patients}

After approval of the present study by our institutional review board, we examined the medical records of 27 consecutive patients with ASD who underwent OLIF with PSF from the thoracic spine to pelvis between January 2015 and December 2018. The indications for the surgery were symptomatic spinal deformity with their sagittal vertical axis (SVA) $\geq 60 \mathrm{~mm}$ or pelvic tilt (PT) $\geq 30^{\circ}$ without vertebral fractures. Data from 27 patients ( 2 men, 25 women) with 75 OLIF levels and a minimum follow-up of 2 years were included. The characteristics of the patients are indicated in Table 1. OLIF was performed for $2.8 \pm 0.4$ spinal levels combined with PSF for $8.3 \pm 0.5$ levels. We examined full-length 36 -inch standing radiographs of both anteroposterior and lateral view obtained before and after surgery every 6 months, until the final follow-up (mean 46.8 months). Additionally, we examined multiplanar CT obtained preoperatively, just after surgery, and 1 year postoperatively. 
Table 1

Patient characteristics

\begin{tabular}{|c|c|}
\hline Number & 27 \\
\hline Age (years) & $70.3 \pm 6.8$ \\
\hline \multicolumn{2}{|l|}{ Sex } \\
\hline Male & 2 \\
\hline Female & 25 \\
\hline Height (cm) & $150.0 \pm 8.7$ \\
\hline Weight (kg) & $53.1 \pm 10.4$ \\
\hline Body Mass Index (kg/m2) & $23.5 \pm 3.5$ \\
\hline Bone density (T-score in lumbar spine) & $-1.9 \pm 1.1$ \\
\hline \multicolumn{2}{|l|}{ Levels for OLIF (cases) } \\
\hline L2/3 & 25 \\
\hline $\mathrm{L} 3 / 4$ & 26 \\
\hline L4/5 & 26 \\
\hline Number of OLIF levels & $2.8 \pm 0.4$ \\
\hline 1 level $(n)$ & 0 \\
\hline 2 levels (n) & 6 \\
\hline 3 levels (n) & 21 \\
\hline Number of levels for posterior fusion surgery & $8.3 \pm 0.5$ \\
\hline Follow-up period (months) & $46.8 \pm 16.6$ \\
\hline \multicolumn{2}{|c|}{$\begin{array}{l}\text { Twenty-seven patients ( } 2 \text { men, } 25 \text { women) with } 75 \text { OLIF levels and a minimum follow-up of } 2 \text { years } \\
\text { were included in this study. OLIF was performed for } 2.8 \pm 0.4 \text { spinal levels combined with PSF for } 8.3 \\
\pm 0.5 \text { levels in patients with mean } 70.3 \text { years of age and }-1.9 \text { of T-score. }\end{array}$} \\
\hline
\end{tabular}

Surgical Methods

OLIF and PSF were performed together or separately as 2-staged surgery. In all patients, OLIF was performed before subsequent PSF. One, 2, or 3-level OLIF from L2 to 5 was performed using a Medtronic OLIF25 Clydesdale Spinal System (Medtronic Sofamor Danek) with the patient in a right lateral decubitus position. A 6 to $10 \mathrm{~cm}$ skin incision was made in the left lateral abdominal region parallel to the fibers of the external oblique muscle. External oblique, internal oblique, and transverse abdominal muscles were then dissected along the direction of their fibers, the retroperitoneal space was accessed by blunt dissection, and the peritoneal content was mobilized anteriorly. The psoas major muscle was identified and reclined posteriorly, and after fixing an OLIF retractor, the annulus fibrosis was exposed for the 
discectomy and to insert the cage. For all 75 segments included in this study, a $6^{\circ}$ lordotic polyetheretherketone cage (OLIF25 Clydesdale Spinal System; Medtronic Sofamor Danek), ranging 8-12 $\mathrm{mm}$ in height, was inserted from the left side of the intervertebral space under guidance from an image intensifier. Allograft bone chips with a diameter of $2-3 \mathrm{~mm}$ produced by our university bone bank were grafted inside the cage [12].

After OLIF surgery, PSF was performed on the same day or 1 week later as second-stage surgery. For all segments with OLIF, total facetectomy, which is equivalent to Grade 2 in the Schwab's spinal osteotomy classification was performed [13]. Additionally, L5-S1 transforaminal lumbar interbody fusion (TLIF) using a Capstone or Capstone Control cage (Medtronic Sofamor Danek) filled with local autograft was performed routinely. Pedicle screws were inserted from the lower thoracic spine to the pelvis, and lumbar lordosis (LL) was increased using a cantilever technique with additional compression using $5.5 \mathrm{~mm}$ diameter rods of titanium-alloy bilaterally. After inserting the rods, laminae were decorticated, and a mixture of local autograft and allograft bone chips was grafted onto the bone surface before closure.

Evaluation of Endplate Injury and Subsidence

All the patients underwent CT at a slice thickness of $0.6 \mathrm{~mm}$ as a part of initial fracture management. CT data were uploaded to picture archiving and communication software (PACS EV Insite, EVIR version 3.6, PSP). The EV Insite software enables calculation of interactively assessable 2-dimensional multiplanar CT reconstructions with adjustable planes. Based on the center position of the cage obtained from axial section of CT data 1 week after surgery, sagittal and coronal sections were reconstructed individually (Fig. 1). The slices of coronal and sagittal sections were reproduced using the CT data obtained before surgery and 1 year after surgery. Endplate fracture was evaluated by CT obtained 1 week after surgery with definition as a displacement of the endplate $\geq 2 \mathrm{~mm}$ compared with the same section from preoperative $\mathrm{CT}$, and subsidence 1 year after surgery evaluated by CT using a definition of displacement of endplate $\geq 2 \mathrm{~mm}$ compared with the displacement 1 week after surgery [14]. Images of the same section at 2 different time points were fused using OsiriX MD software (Pixmeo), and displacement of the endplate was measured separately in sequence (Fig. 2). To specify the location of endplate fracture and subsidence, the endplate was divided into anterior or posterior areas in sagittal section, and right (nonapproach side) or left (approach side) in coronal section from the center line of the cage (Fig. 1). We compared the prevalence of endplate fracture or subsidence between anterior and posterior areas, and between approach and non-approach sides. Additionally, in OLIF segments with intervertebral wedging $\geq 5^{\circ}$ before surgery, the prevalence was also compared between convex and concave sides in coronal section. We similarly compared the prevalence between proximal and distal vertebra of each segment.

Radiological Evaluation of Alignment, Fusion, and Complications

Radiographic measurements including lumbar lordosis (LL, L1-S1), pelvic incidence (PI), PI-LL, pelvic tilt (PT), thoracic kyphosis (TK, T4-T12), sagittal vertical axis (SVA), T1-pelvic angle (TPA), C7-central sacral vertebral line shift (C7-CSVL), and maximum coronal Cobb angle were obtained before surgery, first standing after surgery, and at final follow-up [15-17]. Fusion at 1 year postoperatively was evaluated by 
multiplanar CT and on plain radiographs based on criteria proposed by McAfee modified as follows. (1) Bridging osseous growth between the endplates of the fused vertebral bodies inside or outside of the cage, visible in the plain static radiographs and in CT, and (2) no radiolucent line around the cage visible on CT. (3) No segmental angle change $>5^{\circ}$ between standing and supine positions in plain radiographs [18]. All radiographs were assessed by two independent examiners (spine surgeons), blinded to conditions, and not associated with the surgical procedure. Intra- or postoperative complications were also reviewed to determine their association with endplate fracture, subsidence, alignment and fusion status.

\section{Statistical Analysis}

Statistical analyses were conducted using IBM SPSS Statistics for Windows (version 26). Continuous variables are shown as mean \pm standard deviation (SD) or median value and interquartile range (IQR), and categorical variables are shown as the number and percentage of patients or levels. Continuous variables were compared using an unpaired $t$ test for mean values or a Mann-Whitney $U$ test for median values, and categorical variables were evaluated using a Fisher exact test to compare the frequency of events between groups. $P<0.05$ was considered significant.

\section{Results}

\section{Endplate Fracture}

In the 75 segments that underwent OLIF, endplate fracture was observed at 64 (85.3\%) levels in all 27 patients. In all patients, endplate injury was noted at least one OLIF level. The prevalence of endplate fracture in the posterior area (85.3\%) was significantly higher than that in the anterior area (68.0\%). By contrast, the prevalence in the approach $(77.3 \%)$ and non-approach sides $(81.3 \%)$ was not significantly different (Table 2). A wedging angle $\geq 5^{\circ}$ was observed at $65.3 \%$ of 75 OLIF levels, and among these wedged segments, the prevalence of endplate fracture was $69.4 \%$ on the concave side and $79.6 \%$ on the convex side, but not significantly different. Figure 3 indicates the prevalence of endplate fracture at individual proximal and distal vertebrae. In the sagittal plane, the prevalence of fracture was significantly higher in the posterior area of distal vertebrae (64.0\%), than it was in the anterior area of proximal vertebra $(48.0 \%)$ or distal vertebra $(46.7 \%)$. In the coronal plane, the prevalence of fracture was significantly higher in the distal non-approach side (62.7\%), than it was in the proximal approach side (46.7\%). In 49 wedged segments, the prevalence of fracture was significantly higher in the concave side at a distal level $(71.4 \%)$, than it was in the concave (42.9\%) and convex (38.8\%) sides of proximal vertebra. 
Table 2

Incidence of endplate fracture

Total

$P$ value

\section{Sagittal plane}

Anterior

Posterior

Coronal plane

Left (approach side)

Right (non-approach side)

Concave side

Convex side

The prevalence of endplate fracture in the posterior area was significantly higher than that in the anterior area. By contrast, there was no significant difference in the approach and non-approach sides, and the concave and convex sides.

\section{Subsidence}

Cage subsidence was found in $18.7 \%$ of 75 levels 1 year postoperatively. The prevalence of subsidence subsequent to endplate fracture was $78.6 \%$ of 14 levels. In $82.8 \%$ of 64 levels with endplate fracture, no subsidence was found. As indicated in Table 3, there was no significant difference in the prevalence of subsidence between anterior (8.0\%) and posterior (16.0\%) areas, approach (5.3\%) and non-approach (12.0\%) sides, and concave (12.2\%) and convex (4.1\%) sides. Subsidence occurred with significantly higher prevalence in the non-approach side $(10.7 \%)$ at proximal vertebra than it did in the approach side $(4.0 \%)$ at proximal vertebra (Fig. $4 ; P=0.02$ ). There was no significant difference in prevalence of fracture whether subsidence was subsequent to endplate fracture or not. 
Table 3

Incidence of subsidence

Total

$P$ value

\section{Sagittal plane}

Anterior

$8.0 \%(6 / 75)$

0.21

Posterior

$16.0 \%(12 / 75)$

\section{Coronal plane}

Left (approach side)

$5.3 \%(4 / 75)$

0.25

Right (non-approach side)

$12.0 \%(9 / 75)$

Concave side

$12.2 \%(6 / 49)$

0.27

Convex side

$4.1 \%(2 / 49)$

There was no significant difference in the prevalence of subsidence between anterior and posterior areas, approach and non-approach sides, and concave and convex sides.

Spinopelvic, Global and Local Alignment, and Fusion

Spinopelvic and global alignment was corrected using multiple OLIF combined with PSF (Table 4). Compared with preoperative alignment, LL, PI-LL, PT, SVA, TPA, and Cobb angle were improved significantly after surgery. No parameters changed significantly from just after surgery to the final followup. Segmental lordotic and wedging angles before and after surgery and their changes were not significantly different in OLIF segments with or without endplate fracture (Table 5). Total fusion rate was $81.3 \%$ of OLIF levels at 1 year postoperatively. Neither endplate fracture nor subsidence was associated with the fusion state of affected segments (Table 6). 
Table 4

Spinopelvic alignment

\begin{tabular}{|llll|}
\hline & Pre & Post & Last \\
\hline Lumbar Lordosis (LL) & $2.5[-8.5,15.5]$ & $39.5[33.5,52.5]^{\star}$ & $42[30,52]$ \\
\hline Pelvic Incidence (PI) & $54.5[47.5,58.5]$ & $53.5[47.5,59.5]$ & $53[47,60]$ \\
\hline PI-LL & $49.5[33.5,60]$ & $10[5,23]^{\star}$ & $10[5,23.5]$ \\
\hline Pelvic Tilt (PT) & $39.5[34.5,52]$ & $26[19.5,35]^{\star}$ & $29.5[22,36.5]$ \\
\hline Thoracic Kyphosis (TK) & $28[11.5,36]$ & $35.5[26.5,46]$ & $44[34.5,55]$ \\
\hline Sagittal Vertical Axis (SVA) & $117[86,154]$ & $36[19.5,52.5]^{\star}$ & $66[28.5,97]$ \\
\hline T1 pelvic angle (TPA) & $43[36,53]$ & $22[15.5,28.5]^{\star}$ & $28.5[23,34]$ \\
\hline C7-Central Sacral Vertical Line (CSVL) & $25[13,65]$ & $12[3.5,22.5]$ & $7.5[1.5,27]$ \\
\hline Cobb angle & $32.5[25.5,49]$ & $14[11,18.5]^{\star}$ & $14[10.5,19.5]$ \\
\hline Data are shown as median [1 st quantile, 3rd quantile]. $\square$ P< 0.05 & & \\
\hline $\begin{array}{l}\text { LL, Pl-LL, PT, SVA, TPA, and Cobb angle were improved significantly after surgery } \\
\text { change until the final follow-up. }\end{array}$ & & \\
\hline
\end{tabular}


Table 5

Segmental angle in OLIF levels

\section{Endplate fracture- Endplate fracture+ $P$ value Total}

\begin{tabular}{|c|c|c|c|c|c|}
\hline Number & & 5 & 70 & & 75 \\
\hline Lordotic angle & Pre & $4.1 \pm 3.7$ & $0.37 \pm 8.2$ & 0.23 & $0.59 \pm 8.1$ \\
\hline & Post & $10.7 \pm 2.0$ & $10.1 \pm 4.5$ & 0.59 & $10.0 \pm 4.5$ \\
\hline & Final & $9.3 \pm 3.5$ & $9.9 \pm 4.4$ & 0.74 & $9.8 \pm 4.5$ \\
\hline Wedging angle & Pre & $12.0 \pm 8.2$ & $7.7 \pm 7.2$ & 0.31 & $8.1 \pm 7.3$ \\
\hline & Post & $4.7 \pm 4.1$ & $3.7 \pm 2.9$ & 0.63 & $3.7 \pm 3.0$ \\
\hline & Final & $3.7 \pm 2.9$ & $2.5 \pm 2.2$ & 0.42 & $2.6 \pm 2.7$ \\
\hline$\Delta$ Lordotic angle & Post-Pre & $6.6 \pm 7.0$ & $9.9 \pm 4.4$ & 0.39 & $9.4 \pm 9.0$ \\
\hline & Final-Post & $0.2 \pm 2.4$ & $-0.7 \pm 4.6$ & 0.49 & $0.51 \pm 4.3$ \\
\hline$\Delta$ Wedging angle & Post-Pre & $7.3 \pm 7.0$ & $3.9 \pm 7.2$ & 0.36 & $4.4 \pm 7.3$ \\
\hline & Final-Post & $1.0 \pm 5.9$ & $1.2 \pm 3.6$ & 0.94 & $-1.0 \pm 4.0$ \\
\hline
\end{tabular}

Data are shown as mean \pm S.D.

Segmental lordotic and wedging angles before and after surgery and their changes were not significantly different in OLIF segments with or without endplate fracture.

Table 6

Relationship between fusion status and endplate fracture / subsidence

\begin{tabular}{|c|c|c|c|c|c|}
\hline & & \multicolumn{2}{|c|}{ Fusion } & \multicolumn{2}{|c|}{ Fusion rate } \\
\hline & & - & + & (\%) & $P$ value \\
\hline \multirow[t]{2}{*}{ Endplate fracture } & - & 1 & 4 & 80.0 & \multirow[t]{2}{*}{1.0} \\
\hline & + & 13 & 57 & 81.4 & \\
\hline \multirow[t]{2}{*}{ Subsidence } & - & 11 & 50 & 82.0 & \multirow[t]{2}{*}{0.72} \\
\hline & + & 3 & 11 & 78.6 & \\
\hline Total & & 14 & 61 & 81.3 & \\
\hline
\end{tabular}

Complications 
Besides endplate fracture and subsidence, complications were noted in $59.3 \%$ of patients. The most frequent complication was proximal junctional kyphosis (7 patients), followed by rod fracture (4 patients), deep vein thrombosis (3 patients), and transient thigh numbness and delirium (2 patients). None of these complications were associated with endplate fracture or subsidence. Rupture of anterior longitudinal ligament (ALL) was noted in 4 patients. Among these 4 segments, endplate fracture and subsidence were found in 2 segments and 1 segment, respectively. By contrast, fusion failed in 3 segments, suggesting ALL rupture might be a risk factor for fusion failure.

\section{Discussion}

LLIF is a less invasive method for achieving substantial sagittal and coronal correction with a lower complication rate in ASD surgery [19-22]. Recently, the lordosis distribution index (LDI), or amount of lower arc lordosis (L4 - S1) in proportion to the total lordosis (L1 - S1), is recognized as important for analyzing sagittal malalignment, and LDI should be corrected to between $50-80 \%$, which was classified as aligned $[23,24]$. Ohba et al. retrospectively analyzed the LDI in 57 patients with ASD who had undergone OLIF with PSF, and identified only $67 \%$ of cases that were classified as "aligned", indicating achieving an ideal LDI is sometimes difficult in OLIF with PSF [25]. To achieve an ideal LDI, contouring of rods plays an important role to achieve ideal correction [26-28], but most Japanese patients with ASD are elderly and have osteoporosis as they did in the present study. Even so, surprisingly, $85 \%$ of OLIF levels in all patients showed endplate fracture just after surgery, which is a much higher rate than a Japanese nationwide survey (2.2\%), or meta-analysis (5.26\%) of OLIF $[8,9]$. By contrast, there reported a significant positive correlation between bone mineral density (BMD) and the failure load of vertebrae [29, 30]. In our patients, the mean T-score was -1.9 , indicating most had osteopenia or osteoporosis and this might induce the high prevalence of endplate fracture in the present study.

When the endplate was divided into anterior and posterior portions, the prevalence of endplate fracture was significantly higher in the posterior area (85.3\%) than the anterior area (68.0\%), which is inconsistent with findings of a past study on cadavers, indicating the posterior area is biomechanically stronger than the anterior area [31]. A recent cadaveric study found an endplate injury in $71 \%$ of OLIF segments [32], with a high prevalence similar to that found in the present study under a compression force. This finding supports the hypothesis that a relatively higher prevalence at the posterior area might be related to rod contour, which is shaped for larger lordosis at lower lumbar levels to achieve an ideal LDI, and compressive forces during rodding maneuver using a cantilever technique.

When the prevalence of endplate fracture was compared between proximal and distal vertebra, the prevalence was significantly higher in distal vertebra (Fig. 3). There reported over $90 \%$ of endplate fractures were observed in distal vertebra after LLIF via a transpsoas approach using multiplanar CT [33, 34], as found similarly in the present study. In the coronal plane, prevalence was found highest on the non-approach and concave side, and the wedging angle was corrected from $8.1^{\circ}$ to $3.7^{\circ}$, although the difference between endplate $+/-$ segments was not significant (Table 5). These results suggest that endplate fracture is most likely to occur when expanding the intervertebral space, rather than by direct 
injury from the insertional approach. On the other hand, in cases with degenerative lumbar scoliosis with a mean Cobb angle of $21.1^{\circ}$, the prevalence of endplate fracture was reported less than 20\% [35]. Taken together, the high prevalence we found might be related to the corrective maneuver applied for sagittal correction, which is difficult to avoid to achieve the ideal alignment.

By contrast, the prevalence of cage subsidence at 1 year postoperatively was less in $30 \%$ of cases and $19 \%$ of levels, and subsidence is not directly related to endplate fracture (Fig. 4). Biomechanical studies found that adding an anchor to the ilium reduces mechanical stress. ${ }^{36,37}$ Less impact of endplate fracture on subsequent cage subsidence found in the present study might be due to the biomechanically stable stiffness and reduction of load-sharing established by long fusion from the thoracic spine to pelvis, resulting in a similar fusion rate and sustained alignment correction despite any endplate fracture or subsidence (Tables 5 and 6 ).

There are several limitations to the present study. First, the study design was retrospective, without a control group, and the number of patients was small. Second, we used only one cage design, a $6^{\circ}$ lordotic polyetheretherketone cage. Currently, a OLIF cage can be chosen with a lordotic angle of $0^{\circ}, 6^{\circ}$, or $12^{\circ}$. Choice of another lordotic angle might affect the occurrence of endplate fracture and subsidence.

\section{Conclusions}

A high prevalence of endplate fracture is possibly affected by the corrective maneuver with an ideal rod counter and cantilever force during OLIF corrective surgery for ASD. However, the endplate fracture was affected less on subsequent cage subsidence, fusion status, and sustainment of corrected alignment. Endplate fracture during the OLIF procedure is difficult to avoid, but has little impact on long fusion surgery for ASD, even that performed in elderly patients with osteopenia or osteoporosis.

\section{Abbreviations}

LLIF: lateral lumbar interbody fusion, OLIF: oblique lumbar interbody fusion, ASD: adult spinal deformity, PSF: posterior spinal fusion, BMI: body mass index, CT: computed tomography, SVA: sagittal vertical axis, PT: pelvic tilt, TLIF: transforaminal lumbar interbody fusion, LL: lumbar lordosis, PI: pelvic incidence, PT: pelvic tilt, TK: thoracic kyphosis, TPA: T1-pelvic angle, C7-CSVL: C7-central sacral vertebral line shift, SD: standard deviation, IQR: interquartile range, ALL: anterior longitudinal ligament, LDI: lordosis distribution index, BMD: bone mineral density

\section{Declarations}

Ethics approval and consent to participate

All procedures performed in this study were followed in accordance with the Declaration of Helsinki, and approved by Institutional Review Board (IRB) for Observation and Epidemiological Study in Kitasato university. Under Ethical Guidelines for Medical and Health Research Involving Human Subjects enacted 
by Ministry of Education, Culture, Sports, Science and Technology and Ministry of Health, Labor and Welfare Japan, written informed consent is unnecessary in this study and detailed study design was disclosed on the website of IRB of Kitasato University and the reception of outpatient clinic of Orthopaedic department in Kitasato University Hospital. All participants were given the opportunity of optout provision of their data for 3 months after IRB approval, and informed consent was considered obtained if patients did not provide an opt-out to this study.

Consent for publication

All participants were given the opportunity to opt out of provision of their data if they did not hope the manuscript including their data to be published.

Availability of data and materials

The datasets analyzed during the current study are available from the corresponding author.

Competing interests

The authors declare that they have no competing interests.

Funding

There were no sources of funding for this study.

Contributions

GI, WS, MM \& MT: Conception and design. TI, ES, YM, AK \& YY: Acquisition of data. GI, TA, TN \& KU: Drafting the article. All authors have made substantial contributions to the interpretation of data and the critical revision of the article. All authors have read and approved the final manuscript.

\section{References}

1. Barbagallo GM, Albanese V, Raich AL, et al. Lumbar lateral interbody fusion (LLIF): comparative effectiveness and safety versus PLIF/TLIF and predictive factors affecting LLIF outcome. Evid Based Spine Care J. 2014;5:28-37.

2. Costanzo G, Zoccali C, Maykowski P, et al. The role of minimally invasive lateral lumbar interbody fusion in sagittal balance correction and spinal deformity. Eur Spine J. 2014;23 Suppl 6:699-704.

3. Hijji FY, Narain AS, Bohl DD, et al. Lateral lumbar interbody fusion: a systematic review of complication rates. Spine J. 2017;17:1412-9.

4. Walker CT, Farber SH, Cole TS, et al. Complications for minimally invasive lateral interbody arthrodesis: a systematic review and meta-analysis comparing prepsoas and transpsoas approaches. J Neurosurg Spine. 2019;30:446-60. 
5. Mundis GM, Akbarnia BA, Phillips FM. Adult deformity correction through minimally invasive lateral approach techniques. Spine. 2010;35(26 Suppl):S312-21.

6. Kim KT, Jo DJ, Lee SH, et al. Oblique retroperitoneal approach for lumbar interbody fusion from L1 to S1 in adult spinal deformity. Neurosurg Rev. 2018;41:355-63.

7. Xi Z, Chou D, Mummaneni PV, et al. Anterior lumbar compared to oblique lumbar interbody approaches for multilevel fusions to the sacrum in adults with spinal deformity and degeneration. $J$ Neurosurg Spine. 2020;33:461-70.

8. Fujibayashi S, Kawakami N, Asazuma T, et al. Complications associated with lateral interbody fusion: nationwide survey of 2998 cases during the first 2 years of its use in Japan. Spine. 2017;42:1478-84.

9. Li HM, Zhang RJ, Shen CL. Differences in radiographic and clinical outcomes of oblique lateral interbody fusion and lateral lumbar interbody fusion for degenerative lumbar disease: a metaanalysis. BMC Musculoskelet Disord. 2019;20:582. doi: 10.1186/s12891-019-2972-7.

10. Chang SY, Nam Y, Lee J, et al. Following Single-Level Oblique Lateral Interbody Fusion With Percutaneous Pedicle Screw Fixation. Orthopedics. 2020;43:e283-90.

11. Chung NS, Lee HD, Jeon $\mathrm{CH}$. The Impact of Vertebral End Plate Lesions on the Radiological Outcome in Oblique Lateral Interbody Fusion. Global Spine J. 2020:2192568220941447. doi:

10.1177/2192568220941447. Online ahead of print.

12. Urabe K, Naruse K, Uchino M, et al. The expense for one implantation of a banked bone allograft from a cadaveric donor and the issues affecting current advanced medical treatment in the Japanese orthopaedic field. Cell Tissue Bank. 2009;10:259-65.

13. Schwab F, Blondel B, Chay E, et al. The comprehensive anatomical spinal osteotomy classification. Neurosurgery. 2014;74:112-20.

14. Satake K, Kanemura T, Nakashima H, et al. Cage subsidence in lateral interbody fusion with transpsoas approach: intraoperative endplate injury or late-onset settling. Spine Surg Relat Res. 2017; 1:203-10.

15. Lafage V, Schwab F, Patel A, et al. Pelvic tilt and truncal inclination: two key radiographic parameters in the setting of adults with spinal deformity. Spine. 2009;34:E599-606.

16. Schwab F, Ungar B, Blondel B, et al. Scoliosis Research Society-Schwab adult spinal deformity classification: a validation study. Spine. 2012;37:1077-82.

17. Lafage R, Schwab F, Challier V, et al. Defining Spino-Pelvic Alignment Thresholds: Should Operative Goals in Adult Spinal Deformity Surgery Account for Age? Spine. 2016;41:62-8.

18. McAfee PC. Interbody fusion cages in reconstructive operations on the spine. J Bone Joint Surg Am. 1999;81:859-80.

19. Isaacs RE, Hyde J, Goodrich JA, et al. A prospective, nonrandomized, multicenter evaluation of extreme lateral interbody fusion for the treatment of adult degenerative scoliosis: perioperative outcomes and complications. Spine. 2010;35(26 Suppl):S322-30. 
20. Phillips FM, Isaacs RE, Rodgers WB, et al. Adult degenerative scoliosis treated with XLIF: clinical and radiographical results of a prospective multicenter study with 24-month follow-up. Spine. 2013;38:1853-61.

21. Park HY, Ha KY, Kim YH, et al. Minimally Invasive Lateral Lumbar Interbody Fusion for Adult Spinal Deformity: Clinical and Radiological Efficacy With Minimum Two Years Follow-up. Spine. 2018;43:E813-21.

22. Kanter AS, Tempel ZJ, Ozpinar A, et al. A Review of Minimally Invasive Procedures for the Treatment of Adult Spinal Deformity. Spine. 2016;41 Suppl 8:S59-65.

23. Yilgor C, Sogunmez N, Boissiere L, et al. Global Alignment and Proportion (GAP) Score: Development and Validation of a New Method of Analyzing Spinopelvic Alignment to Predict Mechanical Complications After Adult Spinal Deformity Surgery. J Bone Joint Surg Am. 2017;99:1661-72.

24. Roussouly P and Nnadi C. Sagittal plane deformity: an overview of interpretation and management. Eur Spine J. 2010;19:1824-36.

25. Ohba T, Ebata S, Ikegami S, et al. Indications and limitations of minimally invasive lateral lumbar interbody fusion without osteotomy for adult spinal deformity. Eur Spine J. 2020;29:1362-70.

26. Cidambi KR, Glaser DA, Bastrom TP, et al. Postoperative changes in spinal rod contour in adolescent idiopathic scoliosis: an in vivo deformation study. Spine. 2012;37:1566-72.

27. Wang X, Boyer L, Le Naveaux F, et al. How does differential rod contouring contribute to $3-$ dimensional correction and affect the bone-screw forces in adolescent idiopathic scoliosis instrumentation? Clin Biomech. 2016;39:115-21.

28. Lindsey $C$, Deviren $V, X u Z$, et al. The effects of rod contouring on spinal construct fatigue strength. Spine. 2006;31:1680-7.

29. Belkoff SM, Maroney M, Fenton DC, et al. An in vitro biomechanical evaluation of bone cements used in percutaneous vertebroplasty. Bone. 1999;25(2 Suppl):23S-6S.

30. Fan S, Zhu Q, Wang B, et al. The relationship and clincal significance between bone mineral density and vertebral compressive intensity. Chin J Clin Anat. 2001;1:74-6.

31. Grant JP, Oxland TR, Dvorak MF. Mapping the structural properties of the lumbosacral vertebral endplates. Spine. 2001;26:889-96.

32. Zhang $X, W u H$, Chen $Y$, et al. Importance of the epiphyseal ring in OLIF stand-alone surgery: a biomechanical study on cadaveric spines. Eur Spine J. 2020 doi: 10.1007/s00586-020-06667-2. Online ahead of print.

33. Le TV, Baaj AA, Dakwar E, et al. Subsidence of polyetheretherketone intervertebral cages in minimally invasive lateral retroperitoneal transpsoas lumbar interbody fusion. Spine. 2012;37:1268-73.

34. Malham GM, Parker RM, Blecher CM, et al. Assessment and classification of subsidence after lateral interbody fusion using serial computed tomography. J Neurosurg Spine. 2015;23:589-97.

35. Katz AD, Singh $H$, Greenwood $M$, et al. Clinical and radiographic evaluation of multilevel lateral lumbar interbody fusion in adult degenerative scoliosis. Clin Spine Surg. 2019;32:E386-96. 
36. Casaroli G, Galbusera F, Chande R, et al. Evaluation of iliac screw, S2 alar-iliac screw and laterally placed triangular titanium implants for sacropelvic fixation in combination with posterior lumbar instrumentation: a finite element study. Eur Spine J. 2019;28:1724-32.

37. Cunningham BW, Lewis SJ, Long J, et al. Biomechanical evaluation of lumbosacral reconstruction techniques for spondylolisthesis: an in vitro porcine model. Spine. 2002;27:2321-7.

\section{Figures}
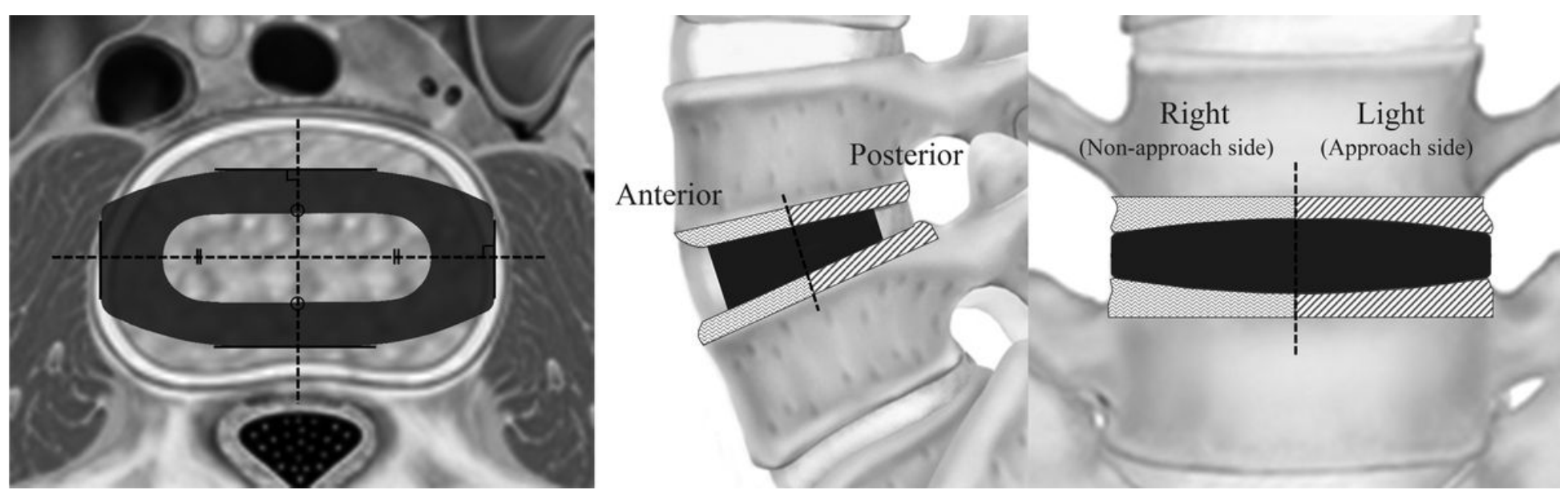

\section{Figure 1}

The vertebral area devided by the cage location Using two-dimensional multiplanar CT reconstructions, CT images were reconstructed parallel to OLIF cage in axial section, and divided into 4 areas based on the center point of the cage (A-Left panel). Vertically to the cage and parallel to the lines in Figure 1A, displacement of endplate was evaluated in sagittal section (B-Middle panel) and coronal section (C-Right panel).

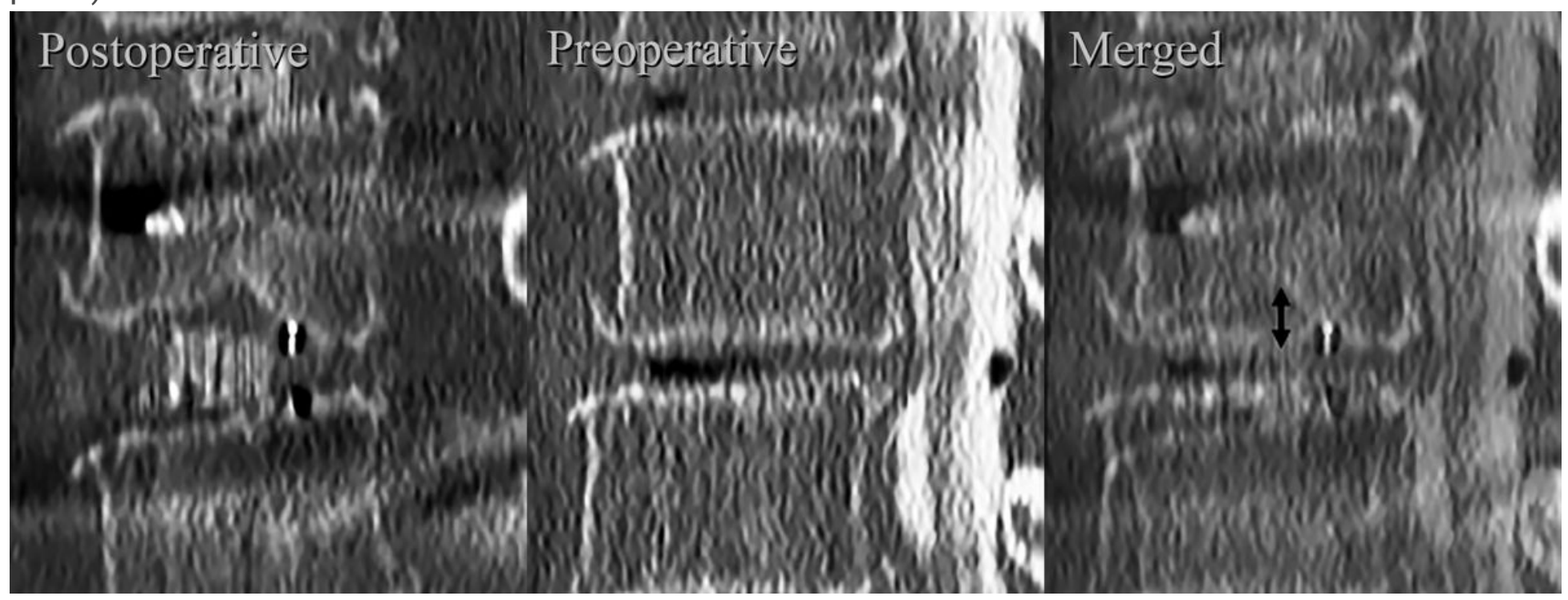

\section{Figure 2}


Displacement of the endplate Images of the same section in sagittal and coronal sections of multiplanar CT at 2 different time points, which are postoperative (A-Right panel) and preoperative (B-middle panel) were merged (C-Right panel), and the minimum distance between endplate was calculated. Displacement $\geq 2 \mathrm{~mm}$ was defined as obvious.

$(\%)$

100

sagittal plane coronal plane

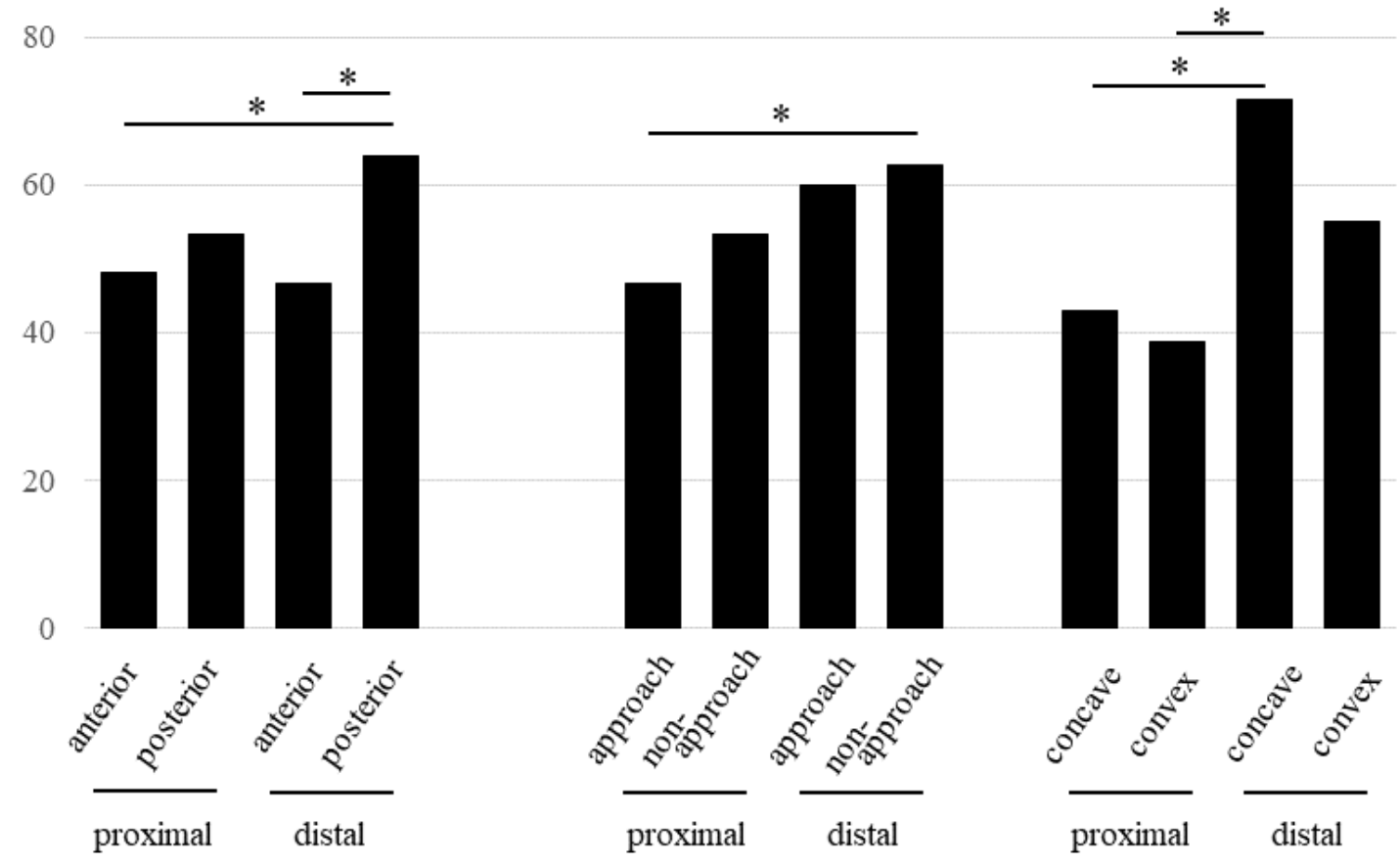

\section{Figure 3}

Prevalence of the endplate fracture Prevalence of the endplate fracture is indicated in percentages. ${ }^{*} \mathrm{P}<$ 0.05 is defined as significant. 
(\%)

20

sagittal section

coronal section

15

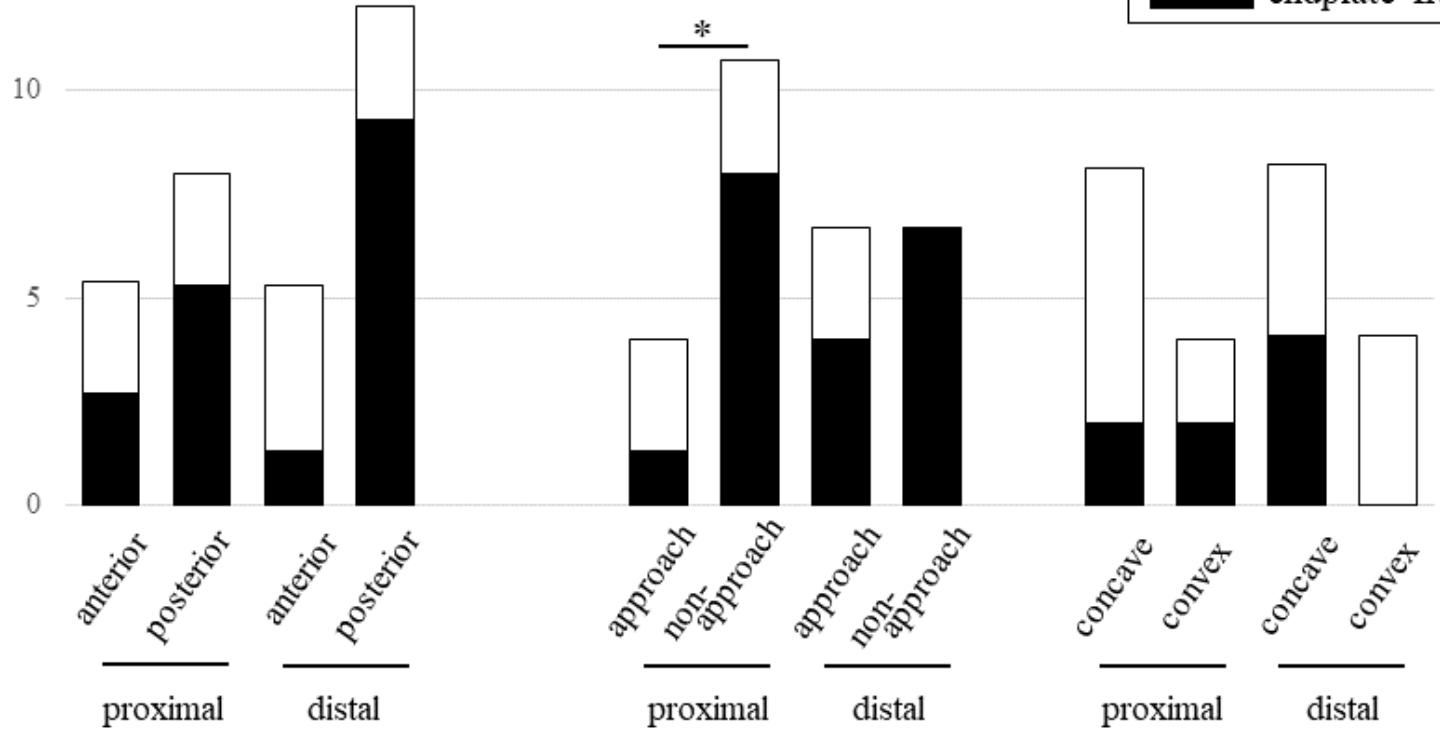

Figure 4

Prevalence of the cage subsidence Prevalence of the cage subsidence is indicated in percentages. White bar: endplate fracture-, black bar: endplate fracture+. ${ }^{\star} \mathrm{P}<0.05$ is defined as significant. 